SHEP-05-15

\title{
Towards a Perfect QCD Gravity Dual
}

\author{
Nick Evans, Jonathan P. Shock, Tom Waterson* \\ School of Physics and Astronomy \\ Southampton University \\ Southampton, SO17 1BJ \\ United Kingdom
}

\begin{abstract}
Many examples of gravitational duals exist of theories that are highly supersymmetric and conformal in the UV yet have the same massless states as $\mathcal{N}=2,1,0$ QCD. We discuss such theories with an explicit UV cutoff and propose that, by tuning higher dimension operators at the cutoff by hand, the effects of the extra matter states in the UV may be removed from the IR physics. We explicitly work in the AdS-Schwarzschild description of $\mathrm{QCD}_{4}$ and tune the operator $\operatorname{Tr} F^{4}$ by relaxing the near horizon limit to reproduce the lattice $0^{++}$glueball mass results. We find that to reproduce the lattice data, the IR and UV cutoffs lie close to each other and there is essentially no AdS-like period between them. The improved geometry gives a better match to the lattice data for $0^{-+}$glueball masses.
\end{abstract}

*evans@phys.soton.ac.uk, jps@phys.soton.ac.uk, trw@phys.soton.ac.uk 


\section{Introduction}

The AdS/CFT Correspondence [1, 2, 3] provides a dual gravitational description of large $N$ $\mathrm{SU}(N), \mathcal{N}=4$ super Yang-Mills theory. The extra fifth non-compact direction of the AdS space corresponds to the energy scale of the gauge theory. Since the gauge theory is conformal, and if it is at strong coupling, the gravity description exists on an infinite line in this fifth direction. Considerable work [4, 5, 6, 7] has been performed on studying the inclusion of relevant operators in the field theory which correspond to fields in AdS whose solutions fall to zero at large radius in AdS. In this way one can study theories that have the massless states of $\mathcal{N}=2,1,0$ theories. At large radius the space returns to $\mathrm{AdS}$ and the gauge theory to the $\mathcal{N}=4$ theory. Typically in the interior the supergravity description becomes singular providing an infra-red block which corresponds to the induced mass gap of the theory with less supersymmetry.

Such constructions have been used as tools to study the low energy behaviour of the $\mathcal{N}=$ 2, 1, 0 gauge theories and are often used for comparison to the pure versions of those theories without the extra states in the UV. Confinement [8, 9, 10] and chiral symmetry breaking [11, 12] are broadly well described. In fact the bound state spectrums of these theories [13, 14, 15, 16, 17, 18, 19] also seem to match well to those observed (and predicted by the lattice) in real QCD. Recently a number of authors have constructed toy phenomenological models of QCD using a slice of AdS space with appropriate fields to describe the low lying hadronic states [20, 21, 22]. These models find good agreement with QCD at the 20-30\% level or better.

Whilst deformed AdS geometries presumably do a good job of catching QCD-like physics in the IR below the mass of the superpartners, these theories all have additional massive states at strong coupling and evolve to a conformal strongly coupled theory in the UV. A priori this appears to leave very non QCD-like theories and any match with QCD states would appear to be telling us mainly about the universality of these masses across a range of gauge theories. In this paper we want to begin addressing the issue of systematically removing this unwanted UV physics. We clearly do not want a large UV strongly coupled conformal interval so we will apply a hard UV cutoff in the gravitational description corresponding to roughly the scale where QCD would transition between perturbative and non-perturbative physics. In the theories developed to date there will be additional fields to those we want even at this scale (typically with masses of order this scale). The couplings of these fields will necessarily alter the physics of the fields we are interested in describing. Since the extra fields are massive we hope that their influence on the running of the gauge coupling will be small. Their main effect will be to distort the coefficients of higher dimension operators in the fields we wish to study. We want to assume that the physics above the cutoff scale is that of QCD rather than the $\mathcal{N}=4$ theory but the higher dimensional operators will be the wrong ones for this case if we just impose a cutoff. The natural correction is to hand tune the higher dimension couplings to the values in QCD to 
reproduce the correct physics. This is what we begin to study in this letter.

The idea of tuning higher dimension operators to remove the effects of "regulator" fields is similar to the idea of perfect or improved actions in lattice gauge theory [23, 24. If working on too coarse a lattice, the lattice infects the QCD physics under study with artifacts. However, in principle, by appropriately tuning the higher dimension operators of the theory it should be possible to precisely reproduce QCD results even on an arbitrarily coarse lattice. In practice, adjusting just one or a few higher dimension operators to correctly reproduce the physical data shows improvement across the whole predicted spectrum.

There are also strong links to the ideas of the exact renormalization group - a number of studies have been made [25, 26] where a gauge theory of interest is UV regulated by either $\mathcal{N}=4$ Yang Mills' conformal nature or by Pauli Villar's type fields. If the mass of these fields is at a scale where there is weak coupling they essentially decouple. However, the exact renormalization group provides a formalism that tracks how one must switch on higher dimension operators to keep the physics invariant even as these fields are made light and brought into the energy regime of strong coupling.

In this paper we will study the glueball mass spectrum of the $\mathrm{AdS}_{7}$ Schwarzschild black hole. This solution describes the theory on the world volume of an M5 brane with a compact dimension and at finite temperature. The resulting theory is believed, below the temperature scale, to describe four dimensional non-supersymmetric Yang Mills theory. The glueball spectrum is well known [13, 14, 15, 16, 17, 18, 19] and lies within 30\% or so of the QCD lattice results. The additional M5 brane fields have masses of order the temperature of the field theory and distort the UV of the theory into a strongly coupled 5+1 dimensional theory. An attempt was made in [14] to remove some of the extra fields in the theory by making the brane configuration rotate. However, to maintain a gravitational description, extra fields are needed to keep the theory at strong coupling in the UV so they can not be removed completely. Instead we will introduce an explicit UV cutoff into this theory and then tune the coefficient of the $\operatorname{Tr} F^{4}$ coupling at the cutoff to try to remove the incorrect UV physics (i.e. the effects of these extra fields) in the glue sector and hence improve the glueball results. To switch on this operator one simply allows the solution to revert to flat space asymptotically by undoing the near horizon limit. The resulting deformation has the correct dimension and symmetry properties to play the role of the $\operatorname{Tr} F^{4}$ coupling. Previous studies of this theory can be found in [27, 28, 29, 30]; the operator may result from any number of changes to the UV of the theory, from switching on gravity to embedding the theory in a multi-centre solution. We imagine that above our UV cutoff the theory is true Yang Mills theory. If we did not impose a cutoff the higher dimension operator would grow into the UV and eventually come to dominate the physics. In this case the operator makes the potential that is responsible for the discrete glueball spectrum unbounded. We therefore take 
the scale where the potential instability sets in as the natural UV cutoff.

We will see that to match the lattice large $N 0^{++}$glueball $^{1}$ mass data, we must make the operator $\operatorname{Tr} F^{4}$ large at a rather low scale. In fact, there turns out to be such a small interval between the UV and IR cutoffs that there is no AdS like geometry left and barely any gravity description at all! This is perhaps not surprising since QCD presumably moves into the strong coupling regime fairly quickly and then almost immediately generates a mass gap. We nevertheless look at the predictions of our short interval for the $0^{-+}$glueballs. Only $N=3$ lattice data exists but our improved geometry is a better match to the data than the unimproved geometry.

\section{The Improved Geometry}

To construct a dual gravitational description of $\mathrm{QCD}_{4}$ we begin with the M5 brane solution of 11d Euclidean supergravity [31].

$$
d s_{11}^{2}=h^{-\frac{1}{3}}\left[\left(1-\frac{b^{6}}{\rho^{3}}\right) d \tau^{2}+\sum_{i=1}^{5} d x_{i}^{2}\right]+h^{\frac{2}{3}}\left[\left(1-\frac{b^{6}}{\rho^{3}}\right)^{-1} d \rho^{2}+\rho^{2} d \Omega_{4}^{2}\right],
$$

where $h$ is the solution of the 5 d Laplace equation and $b$ corresponds to the inverse of the temperature of the dual field theory [32. If we let $\rho=\lambda^{2}$ and go to the non-extremal near horizon limit $\left(b=0, h=\rho^{-3}\right)$ of this metric, we get:

$$
d s_{11}^{2}=\lambda^{2}\left[d \tau^{2}+\sum_{i=1}^{5} d x_{i}^{2}\right]+\frac{4}{\lambda^{2}} d \lambda^{2}+d \Omega_{4}^{2},
$$

ie. $\mathrm{AdS}_{7} \times \mathbf{S}^{4}$ after appropriate scaling of coordinates and a Wick rotation. From this we can see that $\rho$ has mass dimension two.

We now want to modify the metric to include the effect of adding $\operatorname{Tr} F^{4}$ to the dual field theory.

$$
S_{F T}=\int d^{6} x\left[\frac{1}{g^{2}} \operatorname{Tr} F^{2}+G \operatorname{Tr} F^{4}+\cdots\right]
$$

The coupling $G$ has mass dimension -6. The gravitational dual of this can be included by adding a constant term in the solution for $h$

$$
h=\rho^{-3} \rightarrow \rho^{-3}\left(1+\alpha \rho^{3}\right)
$$

i.e. going away from the near horizon limit. $\alpha$ has the right mass dimension -6 to be dual to $G$ plus correctly has no R-charge since it does not depend on angles on the four sphere.

\footnotetext{
${ }^{1}$ Throughout the text we will label the glueballs by their quantum numbers $J^{P C}$
} 
$\mathrm{QCD}_{4}$ is dual to the low energy limit of IIA string theory on the AdS-Schwarzschild background [33, 34] on imposing anti-periodic boundary conditions for the fermions in the compact $\tau$ direction. We can obtain the type IIA metric from (11) by compactifying the $11^{\text {th }}$ dimension and rescaling the metric by a factor $e^{\frac{2 \phi}{3}}=h^{-\frac{1}{6}}$ :

$$
d s_{\mathrm{IIA}}^{2}=h^{-\frac{1}{2}}\left[\left(1-\frac{b^{6}}{\rho^{3}}\right) d \tau^{2}+\sum_{i=1}^{4} d x_{i}^{2}\right]+h^{\frac{1}{2}}\left[\left(1-\frac{b^{6}}{\rho^{3}}\right)^{-1} d \rho^{2}+\rho^{2} d \Omega_{4}^{2}\right] .
$$

This solution has a non-constant dilaton $e^{\phi}=h^{-\frac{1}{4}}$. This is our improved geometry in which we will now calculate the glueball spectra.

Note that the function $h$, being a solution of the five dimensional Laplace equation, can encode a more complicated function if we allow it to have angular dependence. Terms in $h$ that fall off at large radius are associated with operators of the form $\operatorname{Tr} \phi^{n}$ in the field theory [35, 36], whilst those that grow correspond to R-charged higher dimension operator couplings. Since we are interested in the glue sector we will not make use of these operators.

\section{The $0^{++}$Mass Spectrum}

We first calculate the mass spectrum of the $0^{++}$glueball. This is derived on the supergravity side by solving the equation of motion for a massless scalar

$$
\partial_{\mu}\left[\sqrt{g} g^{\mu \nu} e^{-2 \phi} \partial_{\nu} \Phi\right]=0
$$

in the string frame background (5). Assuming that $\Phi$ is of the form $\Phi=f(\rho) e^{i k \cdot x}$ this gives

$$
\frac{1}{\rho} \frac{d}{d \rho}\left[\left(\rho^{4}-\rho\right) \frac{d f}{d \rho}\right]=k^{2}\left(1+\alpha \rho^{3}\right) f(\rho),
$$

where we have set $b=1$ and the mass of the dilaton is $m^{2}=-k^{2}$ in units of $b$.

In order to change this equation of motion into a Schrödinger form, we make a change of the dependant variable to $z$ and rescale $f$

$$
\frac{d z}{d \rho}=\sqrt{\frac{1+\alpha \rho^{3}}{\rho^{3}-1}}, \quad f(z) \rightarrow f(z) e^{-\frac{1}{2} \int d z^{\prime} p\left(z^{\prime}\right)}
$$

where

$$
p(\rho)=\frac{5 \rho^{3}-2+\alpha \rho^{3}\left(8 \rho^{3}-5\right)}{2 \rho \sqrt{\rho^{3}-1}\left(1+\alpha \rho^{3}\right)^{\frac{3}{2}}} .
$$

We now have an equation in Schrödinger form with a potential

$$
-g^{\prime \prime}(z)+Q(z) g(z)=m^{2} g(z), \quad Q(z)=\frac{1}{2} p^{\prime}(z)+\frac{1}{4} p(z)^{2}
$$


or, in terms of the radial coordinate $\rho$

$$
Q(\rho)=\frac{1}{2} \sqrt{\frac{\rho^{3}-1}{1+\alpha \rho^{3}}} \frac{d p}{d \rho}+\frac{1}{4} p(\rho)^{2} .
$$

We plot the potential as a function of $\alpha$ in Figure 1 for different values of $\alpha$. For $\alpha=0$ the pure AdS geometry gives a well that is bounded into the UV and an infinite, discrete glueball spectrum. When $\alpha$ is non-zero, the UV potential is modified and eventually falls to zero. In the field theory the higher dimension operator grows into the UV until it dominates the physics and removes the discrete spectrum. If we allow this to happen then we are not describing a QCD-like theory in the UV, so we will impose a hard UV cutoff, $\Lambda$. The natural scale to place the cutoff is at the turning point of the potential since that includes in the IR theory the highest possible tower of discrete states - we will adopt this value for the cutoff henceforth. Thus as we increase $\alpha$ we will necessarily be working on a shorter radial interval.

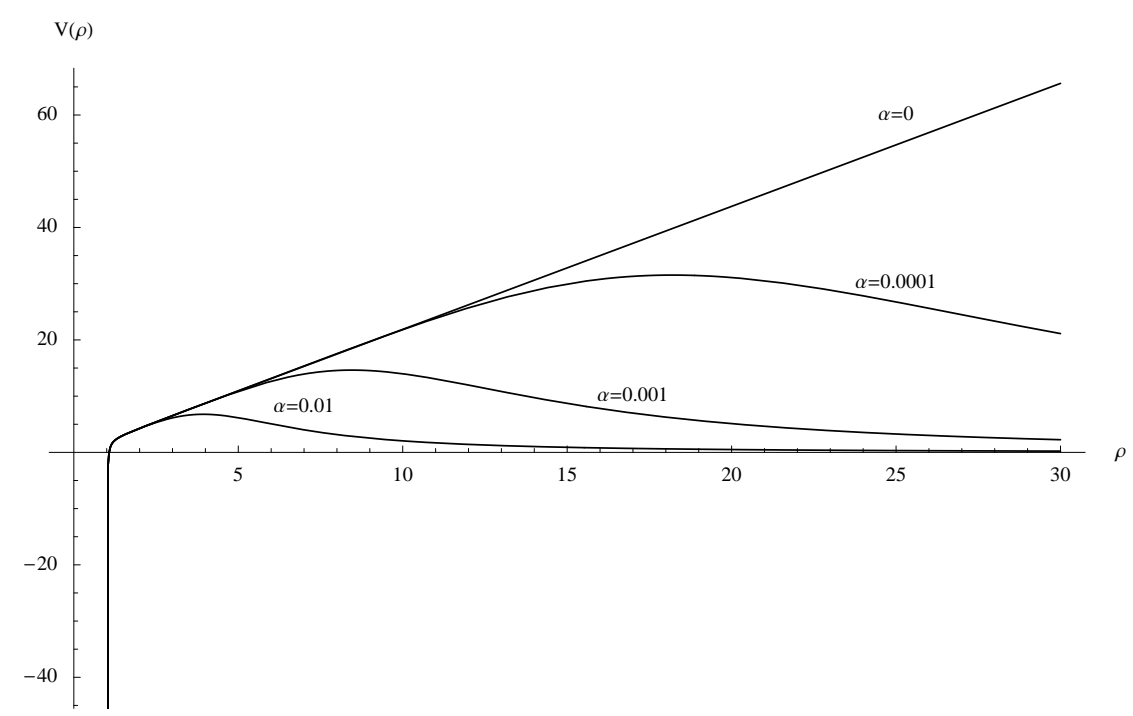

Figure 1: $\mathrm{QCD}_{4}$ Schrödinger potential for the $0^{++}$glueballs $\alpha=0$ to $\alpha=0.01$.

We calculate the eigenvalues of (77) numerically using the "shooting technique", whereby we fix a value for $k^{2}$, set boundary values for $f^{\prime}(\rho)$ at the cutoff $\Lambda$ and then solve the equation of motion numerically down to the black hole horizon at $\rho=1$. We then repeat this for different values of $k^{2}$. The eigenvalues will be those values of $k^{2}$ for which the solution is regular at the horizon i.e. for which $f^{\prime}(0)$ is constant.

We know that for $\alpha=0$ taking $\rho \rightarrow \infty$, the metric goes to AdS space and the normalisable solution to the wave equation (77) goes like $\rho^{-3}$. We will use the naive boundary conditions $f(\Lambda)=\Lambda^{-3}, f^{\prime}(\Lambda)=-3 \Lambda^{-4}$ in all cases below. As $\alpha$ grows and the cutoff falls this boundary condition, which represents the effective dimension of $\operatorname{Tr} F^{2}$, should presumably change - it 
is essentially a matching condition on the dimension that should come from the UV theory. We have checked that if we instead use the boundary conditions $f(\Lambda)=\Lambda^{-(3+\epsilon)}, f^{\prime}(\Lambda)=$ $-(3+\epsilon) \Lambda^{-(4+\epsilon)}$ with $-1<\epsilon<1$ the ratio of the lightest two glueballs masses only changes by $6 \%$. This indicates that the mass spectrum is largely insensitive to the precise values of the boundary conditions. Note that this range includes $\operatorname{Tr} F^{2}$ having dimension four as one might expect in real QCD.

Using this shooting technique, we tune $\alpha$ to get a glueball spectrum that agrees best with the available large $N$ lattice data [37, 38]. Figure 2 shows the ratio $m\left(0^{++*}\right) / m\left(0^{++}\right)$for different values of $\alpha$. We can see that setting $\alpha=0.0855$ gives the correct value for the second glueball mass (the first is fixed by normalisation). This implies that $\Lambda=1.99$ - we will refer to this case as the "improved geometry". For this value of $\alpha$ we get the spectrum of masses shown in Table 1. The glueball masses rise in the theory although we have no more lattice data to compare to for this state.

The result, that to correctly reproduce the lattice data we must raise $\alpha$ so that the theory only provides a description between an IR scale of $b=1$ and a UV scale of $\sqrt{\Lambda}=1.41$ (note $\sqrt{\Lambda}$ has mass dimension 1), is important. Although the original AdS black hole produced results that match the QCD data reasonably we find that to move to a phenomenological model of QCD we must actually distort the AdS space considerably. Indeed, the gravitational theory's interval is worryingly small and non-AdS like. This is not so surprising in terms of real QCD where the regime between the QCD coupling becoming non-perturbative and the scale of the mass gap of the theory is quite small. This result may have important ramifications for attempts to turn toy models of the sort in [20, 21, 22] into true phenomenological tools.

\begin{tabular}{||l|l|l|l|l||}
\hline Glueball State & Improved Geometry & $\alpha=0$ & $N=3$ Lattice & $N=\infty$ Lattice \\
\hline $0^{++}$ & 1.00 & 1.00 & 1.00 & 1.00 \\
\hline $0^{++*}$ & 1.90 & 1.58 & 1.74 & 1.90 \\
\hline $0^{++* *}$ & 3.05 & 2.15 & - & - \\
\hline $0^{++* * *}$ & 4.27 & 2.72 & - & - \\
\hline $0^{++* * * *}$ & 5.52 & 3.33 & - & - \\
\hline
\end{tabular}

Table 1: $\mathrm{QCD}_{4} 0^{++}$glueball masses from AdS $(\alpha=0)$ and Improved $(\alpha=0.0855)$ geometries along with lattice data [37, 38]. Normalisation is such that the ground state mass is set to one.

\section{The $0^{-+}$Mass Spectrum}

We now consider the $0^{-+}$glueballs. We assume that the dominant contribution to their mass spectrum will come from the $\operatorname{Tr} F \tilde{F}$ operator as this is the lowest dimension operator with the 


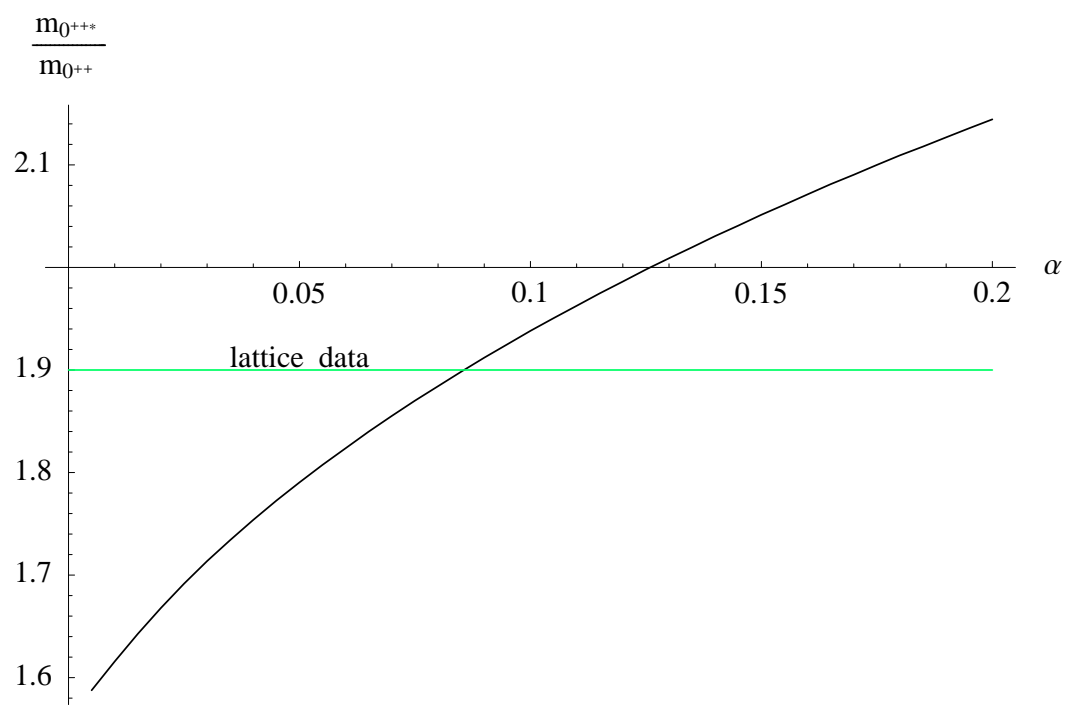

Figure 2: $m_{0^{++*}} / m_{0^{++}}$for different values of $\alpha$

correct quantum numbers. Dual to this on the supergravity side is the RR 1-form $A_{\mu}$. The equation of motion for this is

$$
\partial_{\nu}\left[\sqrt{g} g^{\mu \delta} g^{\nu \sigma}\left(\partial_{\delta} A_{\sigma}-\partial_{\sigma} A_{\delta}\right)\right]=0 .
$$

As with the $0^{++}$, we look for solutions of the form $A_{\tau}=f(\rho) e^{i k \cdot x}$ in the background (5). The result is the equation

$$
\frac{1}{\rho^{4}}\left(\rho^{3}-1\right) \frac{d}{d \rho}\left[\rho^{4} \frac{d f}{d \rho}\right]=k^{2}\left(1+\alpha \rho^{3}\right) f(\rho) .
$$

If we set $\alpha=0.0855$ and $\Lambda=1.99$, which were their optimum values for the $0^{++}$, we get the spectrum shown in Table 2 . We have normalised all masses to the $0^{++}$ground state. The lightest state does not match well to the $\mathrm{N}=3$ lattice data - this state was omitted from the spectrum in [14, 15] which then improves the fit considerably! The effect of our improved geometry is to make the states more massive which improves the fit to the data whether the first state is omitted or left in. To truly match these states to the data would presumably require a higher dimension operator with $P=-1, C=+1$ quantum numbers to be tuned - it is not clear how to include such an operator though.

\begin{tabular}{||l|l|l|l||}
\hline Glueball State & Improved Geometry & $\alpha=0$ & $N=3$ Lattice \\
\hline $0^{-+}$ & 0.35 & 0.29 & 1.61 \\
\hline $0^{-+*}$ & 1.38 & 1.24 & 2.26 \\
\hline $0^{-+* *}$ & 2.48 & 1.84 & - \\
\hline $0^{-+* * *}$ & 3.71 & 2.42 & - \\
\hline
\end{tabular}

Table 2: $\mathrm{QCD}_{4} 0^{-+}$glueball masses from AdS $(\alpha=0)$ and Improved $(\alpha=0.0855)$ geometries along with lattice data [37]. All states are normalised to the $0^{++}$ground state. 


\section{Conclusions}

We have shown that by including the gravitational dual of a higher dimensional field theory operator to the usual AdS-Schwarzschild metric, we can tune our theory to match onto the first excited $0^{++}$glueball state as calculated using lattice techniques. Having fixed the strength of this perturbation, we also find that the $0^{-+}$spectrum is improved. We find that in order to get the correct $0^{++}$spectrum we almost entirely remove the AdS-like region of the space. This ties in with there being only a small energy range between the mass gap and strong coupling region of QCD.

\section{Acknowledgements}

JS and TW are grateful to PPARC for the support of their studentships. NE is grateful to the organisers of the "AdS/CFT and QCD" workshop at Hanyang university in Korea in October 2004 where the idea for this work was first developed.

\section{References}

[1] J. M. Maldacena, "The large N limit of superconformal field theories and supergravity," Adv. Theor. Math. Phys. 2 (1998) 231 [Int. J. Theor. Phys. 38 (1999) 1113] arXiv:hep-th/9711200.

[2] S. S. Gubser, I. R. Klebanov and A. M. Polyakov, "Gauge theory correlators from non-critical string theory," Phys. Lett. B 428 (1998) 105 arXiv:hep-th/9802109.

[3] E. Witten, "Anti-de Sitter space and holography," Adv. Theor. Math. Phys. 2 (1998) 253 arXiv:hep-th/9802150.

[4] L. Girardello, M. Petrini, M. Porrati and A. Zaffaroni, "Confinement and condensates without fine tuning in supergravity duals of gauge theories," JHEP 9905 (1999) 026 arXiv:hep-th/9903026.

[5] L. Girardello, M. Petrini, M. Porrati and A. Zaffaroni, "The supergravity dual of N = 1 super Yang-Mills theory," Nucl. Phys. B 569 (2000) 451 arXiv:hep-th/9909047.

[6] K. Pilch and N. P. Warner, "N = 2 supersymmetric RG flows and the IIB dilaton," Nucl. Phys. B 594 (2001) 209 arXiv:hep-th/0004063.

[7] J. Polchinski and M. J. Strassler, "The string dual of a confining four-dimensional gauge theory," arXiv:hep-th/0003136. 
[8] J. M. Maldacena, "Wilson loops in large N field theories," Phys. Rev. Lett. 80 (1998) 4859 arXiv:hep-th/9803002.

[9] S. J. Rey, S. Theisen and J. T. Yee, "Wilson-Polyakov loop at finite temperature in large N gauge theory and anti-de Sitter supergravity," Nucl. Phys. B 527 (1998) 171 arXiv:hep-th/9803135.

[10] S. J. Rey and J. T. Yee, "Macroscopic strings as heavy quarks in large N gauge theory and anti-de Sitter supergravity," Eur. Phys. J. C 22 (2001) 379 arXiv:hep-th/9803001.

[11] J. Babington, J. Erdmenger, N. J. Evans, Z. Guralnik and I. Kirsch, "Chiral symmetry breaking and pions in non-supersymmetric gauge / gravity duals," Phys. Rev. D 69 (2004) 066007 arXiv:hep-th/0306018.

[12] M. Kruczenski, D. Mateos, R. C. Myers and D. J. Winters, "Towards a holographic dual of large-N(c) QCD," JHEP 0405 (2004) 041 arXiv:hep-th/0311270.

[13] C. Csaki, H. Ooguri, Y. Oz and J. Terning, "Glueball mass spectrum from supergravity," JHEP 9901 (1999) 017 arXiv:hep-th/9806021.

[14] C. Csaki, Y. Oz, J. Russo and J. Terning, "Large N QCD from rotating branes," Phys. Rev. D 59 (1999) 065012 arXiv:hep-th/9810186.

[15] J. A. Minahan, "Glueball mass spectra and other issues for supergravity duals of QCD models," JHEP 9901 (1999) 020 arXiv:hep-th/9811156.

[16] R. de Mello Koch, A. Jevicki, M. Mihailescu and J. P. Nunes, "Evaluation of glueball masses from supergravity," Phys. Rev. D 58 (1998) 105009 arXiv:hep-th/9806125.

[17] R. C. Brower, S. D. Mathur and C. I. Tan, "Glueball spectrum for QCD from AdS supergravity duality," Nucl. Phys. B 587 (2000) 249 arXiv:hep-th/0003115.

[18] H. Boschi-Filho and N. R. F. Braga, "QCD / string holographic mapping and glueball mass spectrum," Eur. Phys. J. C 32 (2004) 529 arXiv:hep-th/0209080.

[19] H. Boschi-Filho and N. R. F. Braga, "Gauge / string duality and scalar glueball mass ratios," JHEP 0305 (2003) 009 arXiv:hep-th/0212207.

[20] J. Erlich, E. Katz, D. T. Son and M. A. Stephanov, "QCD and a holographic model of hadrons," arXiv:hep-ph/0501128.

[21] L. Da Rold and A. Pomarol, "Chiral symmetry breaking from five dimensional spaces," arXiv:hep-ph/0501218. 
[22] G. F. de Teramond and S. J. Brodsky, "The hadronic spectrum of a holographic dual of QCD," arXiv:hep-th/0501022.

[23] P. Hasenfratz and F. Niedermayer, "Perfect lattice action for asymptotically free theories," Nucl. Phys. B 414 (1994) 785 arXiv:hep-lat/9308004.

[24] M. Luscher, "Improved Lattice Gauge Theories," Phys. Lett. 158B (1985) 250.

[25] T. R. Morris, "A gauge invariant exact renormalization group. II," JHEP 0012 (2000) 012 arXiv:hep-th/0006064.

[26] S. Arnone, Y. A. Kubyshin, T. R. Morris and J. F. Tighe, "Gauge invariant regularisation via SU(N|N)," Int. J. Mod. Phys. A 17 (2002) 2283 arXiv:hep-th/0106258.

[27] N. J. Evans, C. V. Johnson and M. Petrini, "Clearing the throat: Irrelevant operators and finite temperature in large N gauge theory," JHEP 0205 (2002) 002 arXiv:hep-th/0112058.

[28] K. A. Intriligator, "Maximally supersymmetric RG flows and AdS duality," Nucl. Phys. B 580 (2000) 99 arXiv:hep-th/9909082.

[29] A. Hashimoto, "Holographic description of D3-branes in flat space," Phys. Rev. D 60 (1999) 127902 arXiv:hep-th/9903227.

[30] N. R. Constable and R. C. Myers, "Exotic scalar states in the AdS/CFT correspondence," JHEP 9911 (1999) 020 arXiv:hep-th/9905081.

[31] G. T. Horowitz and A. Strominger, "Black strings and P-branes," Nucl. Phys. B 360 (1991) 197.

[32] E. Witten, "Anti-de Sitter space, thermal phase transition, and confinement in gauge theories," Adv. Theor. Math. Phys. 2 (1998) 505 arXiv:hep-th/9803131.

[33] O. Aharony, S. S. Gubser, J. M. Maldacena, H. Ooguri and Y. Oz, "Large N field theories, string theory and gravity," Phys. Rept. 323 (2000) 183 arXiv:hep-th/9905111.

[34] N. Itzhaki, J. M. Maldacena, J. Sonnenschein and S. Yankielowicz, "Supergravity and the large N limit of theories with sixteen supercharges," Phys. Rev. D 58 (1998) 046004 arXiv:hep-th/9802042.

[35] P. Kraus, F. Larsen and S. P. Trivedi, "The Coulomb branch of gauge theory from rotating branes," JHEP 9903 (1999) 003 arXiv:hep-th/9811120. 
[36] J. Babington, N. J. Evans and J. Hockings, "Secrets of the metric in $\mathrm{N}=4$ and $\mathrm{N}=2^{*}$ geometries," JHEP 0107 (2001) 034 arXiv:hep-th/0105235.

[37] C. J. Morningstar and M. J. Peardon, "The glueball spectrum from an anisotropic lattice study," Phys. Rev. D 60 (1999) 034509 arXiv:hep-lat/9901004].

[38] B. Lucini and M. Teper, "SU(N) gauge theories in four dimensions: Exploring the approach to $\mathrm{N}$ = infinity," JHEP 0106 (2001) 050 arXiv:hep-lat/0103027. 